\title{
Injectivity Radius and Fundamental Groups of Hyperbolic 3-Manifolds
}

\author{
MATTHEW E. WhITE
}

It is shown that for each integer $n>1$ there exists a constant $R_{n}>0$ such that if $M$ is a closed hyperbolic 3-manifold with Rank $\pi_{1}(M)=n$, then the injectivity radius of $M$ is bounded above by $R_{n}$.

\section{Introduction.}

Thurston's Geometrization conjecture implies that every closed irreducible 3 -manifold with infinite fundamental group containing no $\mathbb{Z} \oplus \mathbb{Z}$ subgroup is hyperbolic. Thus, it is of interest to obtain information about the hyperbolic metric from purely topological data. For example, the Gromov norm, which is defined homologically, turns out to be a certain scalar multiple of volume if the manifold is hyperbolic [Th]. Similarly, there is also an upper bound on the volume in terms of length of any presentation of its fundamental group $[C]$. The injectivity radius of a closed hyperbolic 3-manifold $M$, denoted $\operatorname{inj}(M)$, is one half the minimum length of all essential loops in $M$. This is equivalent to the usual definition from differential geometry (see [DC]). Recall that the rank of a finitely generated group is the minimum number of elements required to generate the group. In this paper we give an upper bound on the injectivity radius in terms of fundamental group rank. It is clear that no such lower bound exists since Dehn-filling of a hyperbolic manifold provides examples of bounded rank with injectivity radius approaching zero. In a subsequent paper, we hope to provide a lower bound on injectivity radius in terms of other group theoretic data. The basic outline of the proof in our present setting is the following: given a closed hyperbolic 3-manifold $M$, we construct a graph of minimal length which carries $\pi_{1}(M)$. By considering the graph's preimage in hyperbolic space, we show that if the injectivity radius is sufficiently large, any relation in the fundamental group forces some of the graph's edges into close proximity. This in turn allows modification of the graph to reduce its length while still carrying fundamental group. We therefore show that, in fact, $\pi_{1}(M)$ is a free group 
of finite rank. A closed hyperbolic 3-manifold is irreducible. Therefore, its fundamental group cannot be a free product. This contradiction proves:

Theorem 4.4. For each integer $n>1$ there exists a universal constant $R_{n}>0$ such that if $M$ is a closed hyperbolic 3-manifold and $\operatorname{Rank} \pi_{1}(M)=$ $n$, then $\operatorname{inj}(M)<R_{n}$.

Residual finiteness of closed hyperbolic 3-manifolds then provides us with:

Corollary 4.5. Given a closed hyperbolic 3-manifold $M$ and an integer $n>0$, there exists a finite sheeted cover

$$
p: \tilde{M} \longrightarrow M
$$

with $\operatorname{Rank} \pi_{1}(\tilde{M}) \geq n$.

We shall use $M$ to denote a closed, connected hyperbolic 3-manifold. In particular, $M$ is isometric to a quotient of $\mathbb{H}^{3}$ by a group of hyperbolic isometries. We say that a connected finite graph $\Gamma$ is an $n$-graph if all vertices of $\Gamma$ are trivalent and $\operatorname{Rank} \pi_{1}(\Gamma)=n$. A carrier $n$-graph for a manifold $M$ is an $n$-graph $\Gamma$ together with a map $f: \Gamma \longrightarrow M$ such that $f_{*}: \pi_{1}(\Gamma) \longrightarrow \pi_{1}(M)$ is an epimorphism. In this case, the length of $\Gamma$ in $M$ is defined by

$$
\ell(f(\Gamma))=\sum_{e \text { an edge of } \Gamma}|e|
$$

where $|e|$ is the length of $e$ measured by pulling back the path metric of $M$ to $\Gamma$. When we refer to a carrier $n$-graph for a given manifold $M$, we implicitly assume that $n=\operatorname{Rank} \pi_{1}(M)$.

This paper is organized as follows: In section 2, we prove some necessary technical results using trivalent graphs. Section 3 contains the $n=2$ case of the main theorem; this was previously known to C. Adams. The main theorem and its corollary are proved in section 4 . We remark here that our results extend naturally to the case of bounded negative curvature.

\section{Trivalent Graphs of Minimal Length.}

Proposition 2.0. For each integer $n>1$, the set of n-graphs is finite. Each n-graph has $3(n-1)$ edges.

Proof. If $\Gamma$ is an $n$-graph, then

$$
\chi(\Gamma)=1-n=(\text { number of vertices })-(\text { number of edges }) .
$$


Note that twice the number of edges equals three times the number of vertices since all vertices of $\Gamma$ are trivalent. Therefore, there are $3(n-1)$ edges.

Let $e_{1} \cdots e_{3 n-1}$ be the oriented edges of $\Gamma$. Given a loop in $\Gamma$, its homotopy class may be represented by a directed edge path. This is a loop in $\Gamma$ which is a product of homeomorphisms $\gamma_{i}:[0,1] \longrightarrow e_{i}$ with the property that for each $i \geq 2$, we have $\gamma_{i-1}(1)=\gamma_{i}(0)$. In our case, we shall also assume that the maps $\gamma_{i}$ are parameterized proportionally to arc length. Although a slight abuse of notation, the standard convention is to denote a directed edge path as

$$
\left(e_{i_{1}}^{ \pm 1}, e_{i_{2}}^{ \pm 1}, \ldots, e_{i_{k}}^{ \pm 1}\right)
$$

with $e_{i}^{-1}$ denoting $e_{i}$ with the opposite orientation. A directed edge path that is a closed loop is called reduced if $e_{i_{m}} \neq e_{i_{m-1}}^{-1}$ for any index $i$ and $e_{i_{1}} \neq e_{n_{k}}^{-1}$. For further details, see $[\mathrm{M}]$.

Proposition 2.1. Let $f: \Gamma \longrightarrow M$ be a carrier n-graph. If $\alpha$ is a simple closed curve in $\Gamma$, then $f_{*}([\alpha]) \neq 0$. In particular, $\ell(f(\alpha))>\operatorname{inj}(M)$.

Proof. Suppose $\alpha$ is a simple closed curve in $\Gamma$. Then $\alpha$ represents a generator of $\pi_{1}(\Gamma)$. Therefore, $f_{*}([\alpha]) \neq 0$ as otherwise we could generate $\pi_{1}(M)$ with fewer than $n$ elements. Moreover, the length of any essential loop in $M$ is at least $2 \operatorname{inj}(M)$.

Lemma 2.2. A closed hyperbolic 3-manifold $M$ has a carrier $n$-graph $\Gamma$ of minimal length. The edges of $\Gamma$ map to geodesic segments in $M$.

Proof. We shall omit much detail since the proof closely follows the standard proof of the existence of closed geodesics in free homotopy classes (see [DC]). Fix $M$ and let $\Gamma$ be an $n$-graph. We may regard $\Gamma$ as a compact subset of $\mathbb{R}^{k}$ with each edge smoothly embedded. Put

$$
d=\inf \left\{\ell(f(\Gamma)) \mid f: \Gamma \longrightarrow M, f_{*} \text { is an epimorphism }\right\} .
$$

Now suppose that $f: \Gamma \longrightarrow M$ is a carrier $n$-graph. Suppose also that there is a loop in $\Gamma$ containing exactly one edge. By Proposition 2.1, the restriction of $f$ to this edge must lift to a path in $\mathbb{H}^{3}$ with distinct endpoints. Therefore, if the restriction of $f$ to an arbitrary edge of $\Gamma$ is not constant, it must lift 
to a path in $\mathbb{H}^{3}$ with distinct endpoints. This means we may construct a family of maps $F=\left\{f_{i}: \Gamma \longrightarrow M\right\}_{i=1}^{\infty}$ such that for each $i \geq 1, f_{i *}$ is an epimorphism, $f_{i}$ is geodesic on each edge of $\Gamma$, and $\ell\left(f_{i}(\Gamma)\right) \longrightarrow d$. Since $M$ is compact, a straightforward Arzela-Ascoli argument then shows that the closure of $F$ is compact in $\mathcal{C}[\Gamma, M]$. Thus, there exists a subsequence $f_{j}$ of $F$ which converges uniformly to a continuous map $f: \Gamma \longrightarrow M$. Uniform convergence implies that $f$ maps every edge to a geodesic segment and that $\ell(f(\Gamma))=d$. We show that $f_{*}$ is an epimorphism. Choose a vertex $x_{0} \in \Gamma$. Given $\epsilon$ small enough, cover each edge of $f(\Gamma)$ with finitely many $\epsilon$ balls such that each is isometric to a ball in $\mathbb{H}^{3}$. Since the convergence of the $f_{j}$ is uniform, by picking $j$ large enough, we can ensure that $f_{j}$ maps into this covering. Now $f_{j *}$ is an epimorphism. Thus, given $[\beta] \in \pi_{1}\left(M, f_{j}\left(x_{0}\right)\right)$, let $[\alpha] \in \pi_{1}\left(\Gamma, x_{0}\right)$ be such that $f_{j *}([\alpha])=[\beta]$. Note that every homotopy class in $\pi_{1}\left(\Gamma, x_{0}\right)$ is represented by a directed edge path in $\Gamma$. Hence, we assume that $\alpha$ is a directed edge path. In each ball, we may homotop $f_{j}$ to $f$. This means $f(\alpha)$ is freely homotopic to $f_{j}(\alpha)$. In particular, we have that $f_{*}$ is an epimorphism. By proposition 2.0, there are finitely many $n$-graphs. Hence we many repeat the above proof for each distinct $n$-graph and then select the graph of minimal length.

Minimal length carrier graphs have a very nice symmetry property which we shall later put to good use.

Definition 2.3. A subset of a hyperbolic 3-manifold is a $\mathbf{Y}$-subset if it is isometric to a subset $\mathcal{S}$ of $\mathbb{H}^{3}$ with the following properties.

(i) $\mathcal{S}$ is composed of three geodesic arcs which meet only at a common endpoint.

(ii) Each pair of geodesic arcs in $\mathcal{S}$ meets the common endpoint with incidence angle $120^{\circ}$.

It follows at once that a $\mathrm{Y}$-subset is planar.

Lemma 2.4. Given a manifold $M$ with $\operatorname{Rank} \pi_{1}(M) \geq 2$, there exists a minimal length carrier n-graph $f: \Gamma \longrightarrow M$ with the following property: each vertex $v$ of $\Gamma$ has a neighborhood $N(v)$ such that $f$ maps $N(v)$ isometrically to a $Y$-subset of $M$. In particular, $f(v)$ is the common endpoint of the geodesic edges in $f(N(v))$. Furthermore, every edge has non-zero length. 
Proof. We assume that $f: \Gamma \longrightarrow M$ is a fixed minimal length carrier $n$ graph with the property that $f$ is an arc-length parameterization on each edge of $\Gamma$. Let $v$ be a vertex of $\Gamma$. Suppose first that no edge of $\Gamma$ has length 0 under $f$. Choose a small neighborhood $W$ of $f(v)$ such that $W$ is isometric to an open ball in $\mathbb{H}^{3}$. The edges of $\Gamma$ incident at $v$ map to geodesic edges in $M$. If any pair of these edges intersect in $W$ at a point other than $f(v)$, then the image of one edge is contained in that of the other edge. It is easy to see that in this case we can modify the graph to reduce its length. Therefore, the images of the three edges intersect only at $f(v)$ in $W$. It also follows that these three edges are planar inside $W$, since otherwise orthogonal projection to a hyperbolic plane reduces the length of $\Gamma$. We now suppose there exists a pair of edges with incidence angle less than $120^{\circ}$. We modify the map $f$ near $v$ as pictured in Figure 2.0. A short hyperbolic trigonometry calculation then shows that this reduces total length.

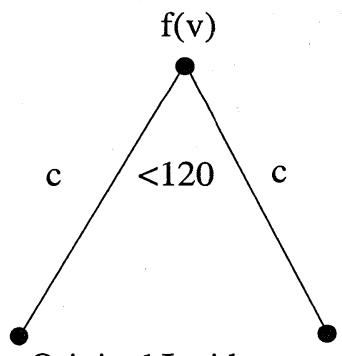

Original Incidence

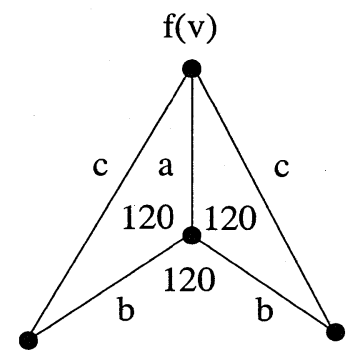

Add Trivalent Edges

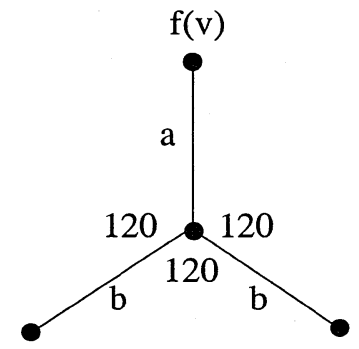

Remove Original Edges

Figure 2.0. Adjustment near a vertex: $2 c>a+2 b$.

Thus, every incidence angle is at least $120^{\circ}$. Of course, the trivalent angle sum is exactly $360^{\circ}$. To complete the proof in the general case, we show that if there exists an edge $e$ of $\Gamma$ with length 0 , then we may modify $f$ to reduce total length. Suppose such an edge $e$ exists. Form a maximal connected subgraph $T$ such that $T$ contains $e$ and $\ell(f(T))=0$. We note that Proposition 2.1 implies that $T$ must be a tree. Hence there exist two vertices $v_{1}$ and $v_{2}$ in $T$ such that $f\left(v_{1}\right)=f\left(v_{2}\right)$ and each vertex has exactly one incident edge of length 0 . 


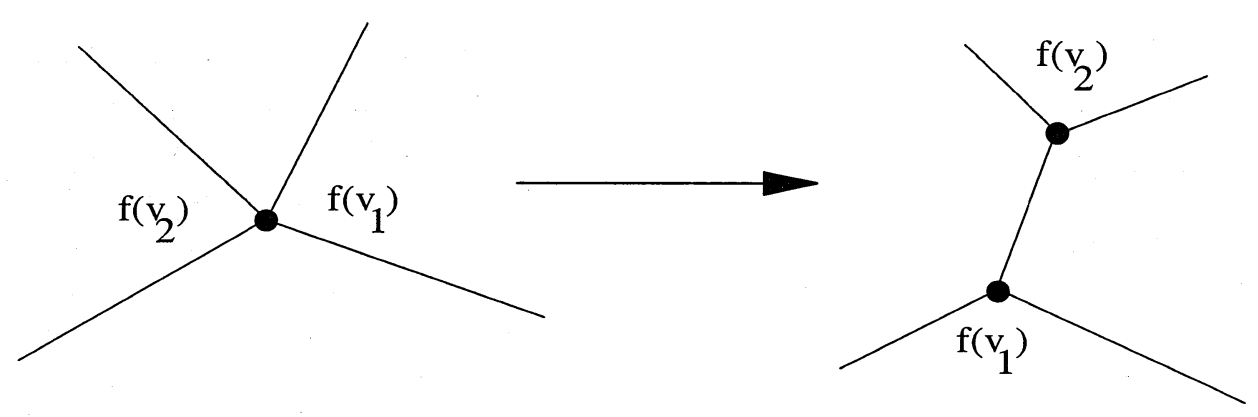

Figure 2.1. Adjustment at identified vertex.

There are two possibilities. In case one, there are four edges incident at $f\left(v_{1}\right)=f\left(v_{2}\right)$. Then we can modify the image of $f$ using the trivalent structure exactly as shown in Figure 2.1. This move separates the vertices by introducing a new edge. The idea is to stretch the edge of length 0 to have small positive length. Then we modify the map using the move in Figure 2.0. This produces a new map of a (possibly different) carrier $n$-graph of strictly lower length. In case two, one of the edges incident at $f\left(v_{2}\right)$ is a subset of an edge incident at $f\left(v_{1}\right)$ and all other incidence angles are $120^{\circ}$. In this case, a short calculation shows that we may reduce length by sliding $f\left(v_{1}\right)$ along the identified edges while straightening the remaining two.

To simplify the exposition, we note the following fact. A minimal length carrier $n$-graph need not be embedded; however, intersections are transverse. Thus, given any $\epsilon>0$ we can adjust the graph by pushing apart intersecting edges inside an $\epsilon$ ball. This allows us to produce an embedded graph with arbitrarily small total increase in length. Of course, some edges will now fail to be geodesic within finitely many $\epsilon$ neighborhoods. But the distortion can be made as small as desired. It is our aim to show that given an integer $n>0$, there is a constant $R_{n}$ such that if a manifold $M$ has $\operatorname{inj}(M)>R_{n}$, then the minimal length carrier $n$-graph $f: \Gamma \longrightarrow M$ provided in Lemma 2.2 has $f_{*}$ an isomorphism. This will imply that $\pi_{1}(M)$ is a free group of rank $n$ which contradicts that $M$ is a closed hyperbolic 3-manifold. To do this, we must prove that $f_{*}$ is injective. Hence, we try to understand the generic properties of a non-trivial class $[\alpha] \in \pi_{1}(\Gamma)$ for which $f_{*}([\alpha])=0$.

Definition 2.5. Fix a universal covering $p: \mathbb{H}^{3} \longrightarrow M$ and let $f: \Gamma \longrightarrow M$ be a minimal length carrier $n$-graph.

(a) We say that $\gamma: S^{1} \longrightarrow \Gamma$ is a compressing loop if $\gamma$ is essential in $\Gamma$ and $f \gamma$ is null homotopic in $M$. 
(b) A shortest compressing loop is a compressing loop of shortest length in $\Gamma$.

(c) A standard lift for kernel $\left(f_{*}\right)$ is a lift to $\mathbb{H}^{3}$ of $f \gamma: S^{1} \longrightarrow M$ where $\gamma: S^{1} \longrightarrow \Gamma$ is a shortest compressing loop.

Remarks. It follows easily that we may represent a shortest compressing loop by a reduced directed edge path. Thus, Lemma 2.4 and the remarks above show we may assume that a standard lift for $\operatorname{kernel}\left(f_{*}\right)$ is a piecewise geodesic embedding of $S^{1}$ in $\mathbb{H}^{3}$ in which the geodesic pieces meet at $120^{\circ}$. angles. We shall call the geodesic pieces in the image of a standard lift edges. Notice that these are lifted edges of the minimal length carrier graph. We now abstract the important properties of standard lifts.

Definition 2.6. A path $h:[0,1] \longrightarrow \mathbb{H}^{3}$ is a Geodesic-120 path if the following conditions hold:

(i) $h$ is a (possibly closed) piecewise geodesic path.

(ii) edges in the image of $h$ meet at $120^{\circ}$ angles.

The key point is that any standard lift we construct for a given closed hyperbolic 3-manifold will be a closed geodesic- 120 path in $\mathbb{H}^{3}$. For our purposes, it will be helpful to think of the image of a standard lift as a tractable geometric object in $\mathbb{H}^{3}$. Frequently, we shall also need to consider an important type of subpath in a geodesic-120 path.

Definition 2.7. Let $h:[0,1] \longrightarrow \mathbb{H}^{3}$ be a geodesic-120 path. Let $[a, b] \subset$ $[0,1]$ where $h(a)$ and $h(b)$ are endpoints of edges in $H=h([0,1])$. A segment of $h$ joining $h(a)$ to $h(b)$ is a subpath $s=\left.h\right|_{[a, b]}:[a, b] \longrightarrow \mathbb{H}^{3}$.

Naturally, if a geodesic-120 path is an embedding, given two endpoints there is exactly one segment joining them.

Lemma 2.8. Let $f: \Gamma \longrightarrow M$ be a minimal length carrier n-graph. If $h: S^{1} \longrightarrow \mathbb{H}^{3}$ is a standard lift, then in any segment of $h\left(S^{1}\right)$ containing at least $3(n-1)$ edges, there is a edge with hyperbolic length $\geq \frac{\operatorname{inj}(M)}{3(n-1)}$.

Proof. Given a manifold $M$, Proposition 2.0 shows that an $n$-graph $\Gamma$ in $M$ has $3(n-1)$ edges. Thus, a simple closed curve in $\Gamma$ has less than $3(n-1)$ edges. By Proposition 2.1, the length of such a curve is at least inj $(M)$. Now 
since $h$ is a standard lift, $h=f \gamma$ where $\gamma$ is a shortest compressing loop. We consider how $\gamma$ behaves in $\Gamma$. We may view $\gamma$ as a product of directed closed edge paths $\alpha_{1} \alpha_{2} \ldots \alpha_{k}$ with the following properties: (a) each path $\alpha_{i}$ has at most $3(n-1)$ edges (b) each path $\alpha_{i}$ contains an edge $e$ such that the path defined by removing the edge $e$ from $\alpha_{i}$ is not a loop (c) each path $\alpha_{i}$ contains a simple closed curve with at most $3(n-1)$ edges. Thus, each $\alpha_{i}$ contains an edge of length at least $\frac{\operatorname{inj}(M)}{3(n-1)}$. The proof is complete since this argument is independent of the starting vertex for the path $\gamma$.

\section{The Case Rank $\left(\pi_{1}(M)\right)=2$.}

In the remainder of this paper, we use the Poincare Disc model for $\mathbb{H}^{3}$. We shall also let $\mathcal{W}$ denote the horoball with diameter given by the geodesic joining 0 to $(-1,0,0)$. In this section, we shall prove the main theorem in the special case of Rank $\pi_{1}(M)=2$. Note that there are precisely two 2-graphs (see Figure 3.0).
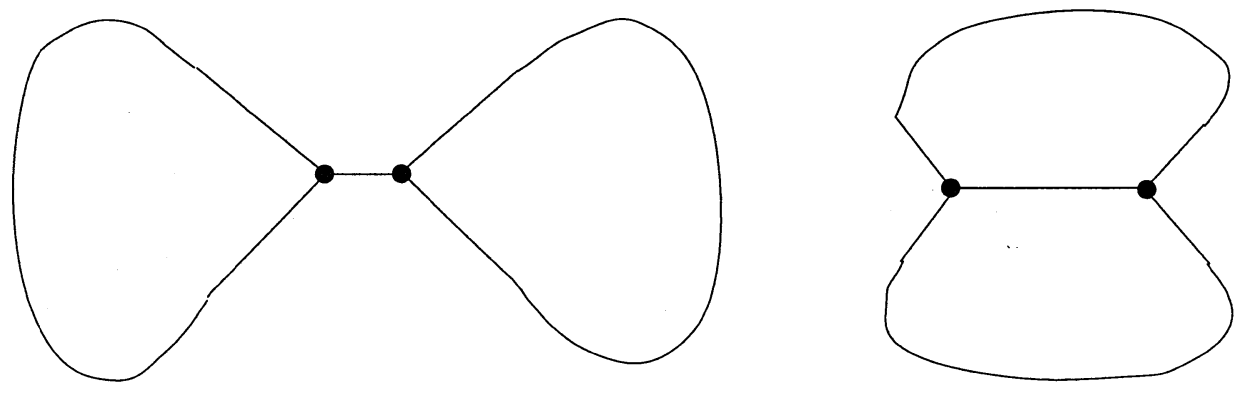

Figure 3.0. The two possible 2-graphs.

Lemma 3.0. There exists $R_{2}>0$ such that if $M$ is a closed, connected, hyperbolic 3-manifold with $\operatorname{Rank} \pi_{1}(M)=2$ then $\operatorname{inj}(M) \leq R_{2}$.

Proof. The proof is established by considering Geodesic- 120 paths in $\mathbb{H}^{3}$. Given a closed, connected hyperbolic 3-manifold $M$ and a minimal length carrier 2-graph $f: \Gamma_{2} \longrightarrow M$ we may choose a standard lift $h: S^{1} \longrightarrow \mathbb{H}^{3}$ for kernel $\left(f_{*}\right)$. Let $H$ denote $h\left(S^{1}\right)$. Since $H$ is compact, there exist points $v, w \in H$ of maximal distance apart. By an isometry of $\mathbb{H}^{3}$, we may assume that $w=0$ and that $v$ lies on the geodesic joining 0 to $(-1,0,0)$. Now $H \subset \bar{B}(v, d(v, 0))$ which implies that $H$ is contained in the horoball $\mathcal{W}$. 


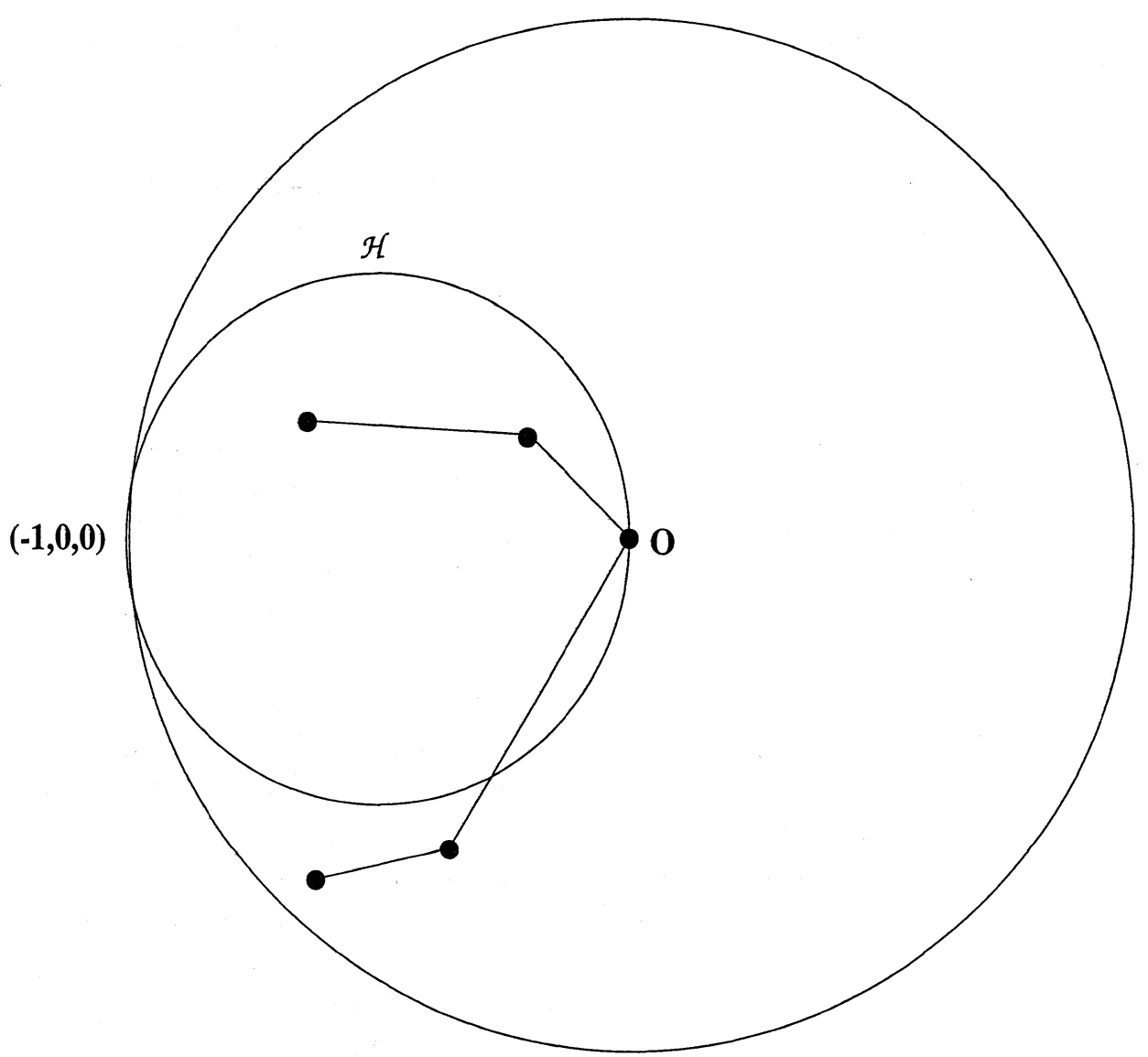

Figure 3.1. Escape of Standard Lift in Rank 2 Case.

Note also that since $H$ is a Geodesic- 120 path, 0 and $v$ must be endpoints of edges of $H$. Moreover, since $H$ is a loop, there must be two edges of $H$ incident at 0 . Consider these two edges. Geodesics through 0 in the disc model correspond to Euclidean diameters of the unit ball in $\mathbb{R}^{3}$. Since the angle between the edges is $120^{\circ}$, there exists $L_{0}>0$ so that if one of the edges of $H$ incident at 0 has length at least $L_{0}$, then $H$ is not contained in $\mathcal{W}$. There are precisely two 2-graphs (see Figure 3.0) and each of these graphs has three edges. By using Proposition 2.1, we see that a 2-graph may have at most one "short" edge. More precisely, if $\operatorname{inj}(M)>2 L_{0}$, then the image $H$ of any standard lift has the following property: given any pair of edges of $H$ with a common endpoint, at least one edge has length greater than $L_{0}$. In other words, put $R_{2}=2 L_{0}$. If inj $(M)>R_{2}$, (after isometry of $\left.\mathbb{H}^{3}\right)$ any standard lift for kernel $\left(f_{*}\right)$ must have a edge which is not contained in $\mathcal{W}$. This contradiction shows that $\operatorname{kernel}\left(f_{*}\right)$ is trivial, so that $\pi_{1}(M)$ is 
actually a free group of rank 2 . Since this is impossible, we conclude that $\operatorname{inj}(M) \leq R_{2}$.

Remark. It is possible to explicitly compute an upper bound for injectivity radius in the $n=2$ case. One finds using a very simple geometry calculation that $L=2.6338 \ldots$ so that $\operatorname{inj}(M) \leq 5.2676 \ldots$.

\section{Proof of Main Theorem.}

The general case is more subtle because $n$-graphs with $n>2$ have more edges. Although large injectivity radius will ensure that minimal length carrier graphs have some long edges, if $n>2$ these graphs may have subpaths of very short edges. This prevents us from using the simple geometric approach in Lemma 3.0 since the image of a standard lift can have very large geodesic edges while remaining inside the horoball $\mathcal{W}$. Instead, we will argue that, for sufficiently large injectivity radius, if the standard lift is contained in $\mathcal{H}$, then it is possible to reduce the length of the minimal length carrier $n$-graph. We will produce a short cut arc in $\mathbb{H}^{3}$ which allows us to make a shorter carrier graph using "cut-and-paste" in the manifold below. To do this, we need two technical lemmas which we now describe. Recall that $\mathbb{H}^{3}$ enjoys "thin triangles." This means there is a universal constant $\Delta$, the thin triangles constant, such that given a hyperbolic triangle with geodesic sides $X, Y$ and $Z$ and a point $x \in X$, there exists a point $y \in Y \cup Z$ with $d(x, y)<\Delta$. The idea is to use this fact together with the geometry of the horoball to show that sufficiently large injectivity radius forces a standard lift to be "thin." Our first lemma is motivated by this fact:

Proposition 4.0. Given $L>0$, if $M$ is a closed hyperbolic 3-manifold with $n=\operatorname{Rank}\left(\pi_{1}(M)\right)$, and $\operatorname{inj}(M)>[3(n-1)]^{2} L$, then every standard lift for $\pi_{1}(M)$ has two edges of length at least $L$.

Proof. Let $M$ satisfy the hypothesis, and let $h: S^{1} \longrightarrow \mathbb{H}^{3}$ be a standard lift with image $H$. Lemma 2.8 shows that any standard lift has one edge of length at least $3(n-1) L$. But if $H$ has exactly one edge of length at least $3(n-1) L$, then it has at most $3(n-1)$ edges. Therefore, $H$ cannot be a closed loop in $\mathbb{H}^{3}$ unless there is another edge of length at least $L$.

This proposition suggests we should try to produce our short cut arc between two "long" edges. The lemma below says that, if $A$ and $B$ are two such edges 
in $\mathcal{W}$ that start at points "close" to the origin, then there is a short cut arc between them.

Lemma 4.1 (Short Cut Lemma). For any $\delta>0$ there exists $\bar{L}(\delta)>0$ such that if $A=\left[a, a^{\prime}\right]$ and $B=\left[b, b^{\prime}\right]$ are geodesic edges contained in $\mathcal{W}$ with:

(i) $a, b \in \bar{B}(0, \delta)$,

(ii) $|A|,|B| \geq \bar{L}$,

then there exist points $e \in A$ and $f \in B$ such that:

(i) $d(e, a)<\frac{1}{3} \bar{L}$ and $d(f, b)<\frac{1}{3} \bar{L}$

(ii) $d(e, f)<d(e, a)-\Delta$ and $d(e, f)<d(f, b)-\Delta$.

Proof. See Figure 4.0. Given $A$ and $B$ as in the hypothesis, let $l_{A}=\left[0, a^{\prime}\right]$, $l_{B}=\left[0, b^{\prime}\right]$, and $\phi$ be the angle between $l_{A}$ and $l_{B}$. Let $\delta>0$. Choose $L_{1}>2(\delta+\Delta)$. This choice assures that given $A, B$ as in hypothesis with $|A|,|B|>L_{1}$, we have $a^{\prime}, b^{\prime} \notin \bar{B}(0, \delta)$. Now notice that as $L_{1} \rightarrow \infty$, we have for all $A, B$ as in the hypothesis with $|A|,|B|>L_{1}$, that $a^{\prime}, b^{\prime} \rightarrow(-1,0,0)$ in the Euclidean metric. Hence, we may choose $L_{2}>L_{1}$ so that given any $A$ and $B$ as in the hypothesis with $|A|,|B|>L_{2}$, we are guaranteed to have $\left|l_{A}\right|,\left|l_{B}\right|$ sufficiently large and $\phi$ sufficiently small that there exist points $x \in l_{A}$ and $y \in l_{B}$ with $d(x, \partial \bar{B}(0, \delta)), d(y, \partial \bar{B}(0, \delta))=5 \Delta$ and $d(x, y)<\Delta$. Using thin triangles, there exist points $e \in A$ and $f \in B$ such that $d(e, x), d(f, y)<\Delta$. This implies $d(e, f)<3 \Delta$. Now choose $\bar{L}(\delta)=3 \max \left(L_{2}, 6 \Delta+2 \delta\right)$. Then $d(e, a), d(f, b)>4 \Delta$ which proves that $d(e, f)<d(e, a)-\Delta$ and $d(e, f)<d(f, b)-\Delta$. Also, $d(e, a) \leq 6 \Delta+2 \delta$ so that $d(e, a)<\frac{1}{3} \bar{L}(\delta)$.

Remark. In subsequent work, we shall cut out one of the geodesics $[e, a]$ or $[f, b]$ and paste in $[e, f]$. It is worth mentioning at this point that conclusion (ii) tells us $\ell([e, a])-\ell([e, f])>\Delta$ and $\ell([f, b])-\ell([e, f])>\Delta$, so the short cut reduces length by at least $\Delta$. The small perturbation needed to make $\Gamma$ embedded can be chosen to change lengths less than $\Delta$. 


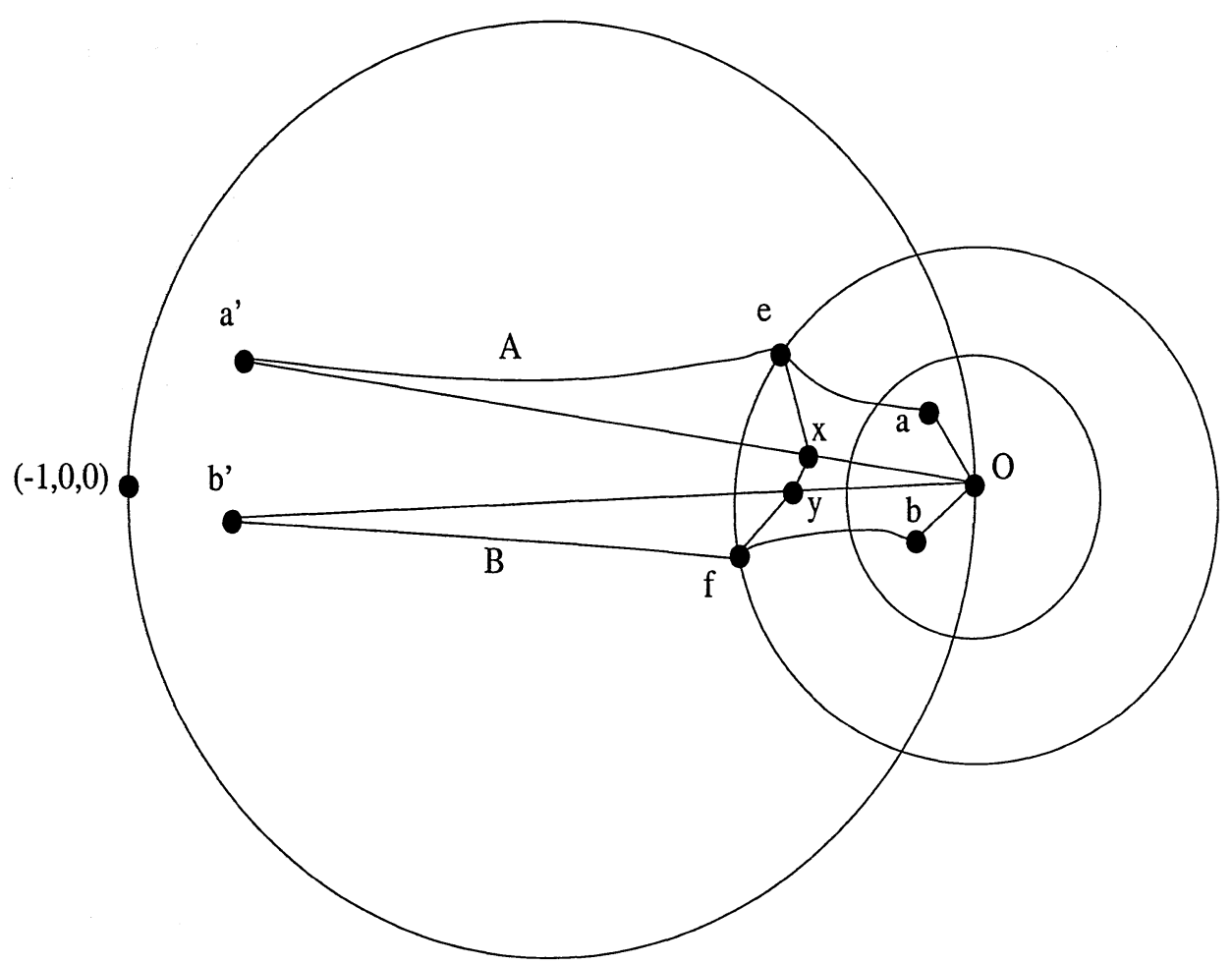

Figure 4.0. Trapping of geodesic segments.

The next two results show that we can actually achieve the conditions in the hypothesis of Lemma 4.1 for embedded Geodesic- 120 paths provided we attach the right assumptions. Suppose that $n=\operatorname{Rank}\left(\pi_{1}(M)\right)$. If $\operatorname{inj}(M)$ is very large, Lemma 2.8 and Proposition 4.0 imply that there are two "very long" edges in a standard lift for $\pi_{1}(M)$. Denoting these two edges $A$ and $B$, we then notice that by Lemma 2.8 there is a Geodesic-120 path of at most $6 n-6$ edges joining $A$ to $B$. We show that either the starting points of $A$ and $B$ are sufficiently close or there is another (not quite as) "long" edge between $A$ and $B$. Taking innermost "long" edges then provides the requirement for the short cut lemma. The basis for the procedure is the following fact about finite sequences of numbers:

Proposition 4.2. Let $L(0)<L(1)<L(2)<\ldots$ be a given sequence of positive numbers. Let $x_{0}, \ldots x_{n}$ and $y_{0}, \ldots y_{m}$ be sequences of positive numbers with $k=n+m$ such that:

(i) $x_{n}$ and $y_{m}$ are both greater than $\max \left\{x_{n-1}, \ldots, x_{0}, y m-1, \ldots, y_{0}\right\}$. 
(ii) $x_{n}$ and $y_{m}$ are both greater than $L(k)$.

Then one of the following conditions must hold:

(1) There exist $r \geq 0$ and $s \geq 0$ such that for $q=r+s$,

(i) $x_{r}$ and $y_{s}$ are both greater than $\max \left\{x_{r-1}, \ldots, x_{0}, y s-1, \ldots, y_{0}\right\}$.

(ii) $x_{r}$ and $y_{s}$ are both greater than $L(q)$

(iii) $\sum_{i=0}^{r-1} x_{i}+\sum_{i=0}^{s-1} y_{i} \leq q L(q-1)$.

(2) Either $x_{0}>L(0)$ or $y_{0}>L(0)$.

Proof. Let $x_{0}, \ldots x_{n}$ and $y_{0}, \ldots y_{m}$ be sequences which satisfy the hypothesis. We may assume that $n \geq 1$ and $m \geq 1$ as otherwise (2) holds. We may also assume that $\sum_{i=1}^{n} x_{i}+\sum_{i=1}^{m} y_{i}>k L(k-1)$. Then without loss of generality there exists $x_{r}$ with $r<n$ such that $x_{r}>L(k-1)$. By choosing $s=m$ if necessary, there also exists $y_{s}>L(k-1)$. We choose $r$ and $s$ to be as small as possible. If $r=0$ or $s=0$, the proof is finished since (2) holds. Otherwise we have that $x_{r}$ and $y_{s}$ are both greater than $\max \left\{x_{r-1}, \ldots, x_{0}, y s-1, \ldots, y_{0}\right\}$. Note also that $r+s \leq n-1+m=k-1$. Hence, for $q=r+s$ we have $x_{r}>L(k-1) \geq L(q)$ and $y_{s}>L(k-1) \geq L(q)$. Now if 1(iii) holds, the proof is done. Suppose otherwise. Repeat the above procedure on the sequences $x_{r}, \ldots x_{n}$ and $y_{s}, \ldots y_{m}$; continuing in this manner, we either obtain sequences $x_{r^{\prime}}, \ldots x_{0}$ and $y_{s^{\prime}}, \ldots y_{0}$ which satisfy (1) or we run out of sequence elements so that we obtain (2).

Lemma 4.3. For each integer $k \geq 0$ there is an $L(k)>0$ such that if $g:[0,1] \longrightarrow \mathbb{H}^{3}$ is an embedded Geodesic-120 path with $G=g([0,1])$ which satisfies:

(i) $0 \in G$

(ii) There exist two geodesic edges $A$ and $B$ in $G$ with $|A|,|B|>L(k)$

(iii) Let $S$ be the segment of $g$ which joins $A$ to $B$. Then $0 \in S$ and $S$ has at most $k$ edges.

(iv) Every edge of $S$ has length at most $L(k)$. 
Then one of the following holds:

(1) $G$ is not contained in the horoball $\mathcal{W}$.

(2) There exist edges $E_{1}$ and $E_{2}$ in $G$ such that:

(i) $g^{-1}\left(E_{1}\right)$ and $g^{-1}\left(E_{2}\right)$ are in different components of $[0,1]-$ $g^{-1}(0)$.

(ii) Every edge in the segment joining $E_{1}$ to 0 has length less than $\left|E_{1}\right|$ and $\left|E_{2}\right|$.

(iii) Every edge in the segment joining $E_{2}$ to 0 has length less than $\left|E_{1}\right|$ and $\left|E_{2}\right|$.

(iv) Let $e_{1} \in E_{1}$ and $e_{2} \in E_{2}$ be the endpoints of these edges contained in the segment joining $E_{1}$ to $E_{2}$. Then there is a short cut arc $F$ joining $e \in E_{1}$ to $f \in E_{2}$ such that $d\left(e_{1}, e\right)>|F|+\Delta, d\left(e_{1}, e\right)<$ $\frac{1}{3} L(k)$, and $d\left(e_{2}, f\right)<\frac{1}{3} L(k)$.

Proof. Define the following sequence: set $L(0)$ equal to the $L_{0}$ in the proof of Lemma 3.0. Recall that this is the length required to force one of the edges at 0 to leave the horoball $\mathcal{W}$. Put $L(1)=2 L(0)$ and for $k \geq 2$ define $L(k)=\bar{L}(k L(k-1))$ with $\bar{L}$ provided by Lemma 4.1. This implies that for $k \geq 2, L(k) \geq 2(k L(k-1)+\Delta)$ so that evidently $L(0)<L(1)<L(2) \ldots$ is an increasing sequence of positive numbers. Now fix $k \geq 0$ and let $G$ be the image of a geodesic-120 path which satisfies the hypothesis. Let $x_{n}=|A|, y_{m}=|B|$, and let $x_{n-1}, \ldots x_{0}$ denote the lengths of the edges joining $A$ to 0 labelled in decreasing order as we proceed from $A$ to 0 . Likewise, let $y_{m-1}, \ldots y_{0}$ denote the lengths of the edges joining $B$ to 0 . By hypothesis (iii), $n+m=k_{1} \leq k$. By (ii), $x_{n}>L(k) \geq L\left(k_{1}\right)$ and $y_{m}>L(k) \geq L\left(k_{1}\right)$. Also, by (iv) we have that $x_{n}$ and $y_{m}$ are both greater than $\max \left\{x_{n-1}, \ldots, x_{0}, y m-1, \ldots, y_{0}\right\}$. This means these sequences satisfy the hypothesis of Proposition 4.2. Conclusion (2) of Proposition 4.2 gives that $x_{0}>L(0)$ or $y_{0}>L(0)$. This means that either $x_{0}$ or $y_{0}$ leaves the horoball $\mathcal{W}$; hence, $G$ is not contained in $\mathcal{W}$. Otherwise, conclusion (1) of Proposition 4.2 gives us subsequences $x_{r}, \ldots x_{0}$ and $x_{s}, \ldots, x_{0}$ with these properties: $x_{r}>L(q), y_{s}>L(q), x_{r}$ and $y_{s}$ are both greater than $\max \left\{x_{r-1}, \ldots, x_{0}, y s-1, \ldots, y_{0}\right\}$, and $\sum_{i=0}^{r-1} x_{i}+\sum_{i=0}^{s-1} y_{i} \leq q L(q-1)$. Refer to Figure 4.1. Geometrically, this means the following. Let $E_{1}$ and $E_{2}$ be the edges of $G$ which correspond respectively to the lengths $x_{r}$ and $y_{s}$. It follows at once that statements 2(i) through 2(iii) hold. To see that 2(iv) is 
also true, note that the segment joining $E_{1}$ to 0 has length at most $q L(q-1)$ since $\sum_{i=0}^{r-1} x_{i} \leq q L(q-1)$. The analogous statement holds for the segment joining $E_{2}$ to 0 . Thus, $e_{1}, e_{2} \in \bar{B}(0, q L(q-1))$. Since $\left|E_{1}\right|=x_{r}>L(q)$ and $\left|E_{2}\right|=y_{s}>L(q)$, Lemma 4.1 applies to give us the required short cut arc F.

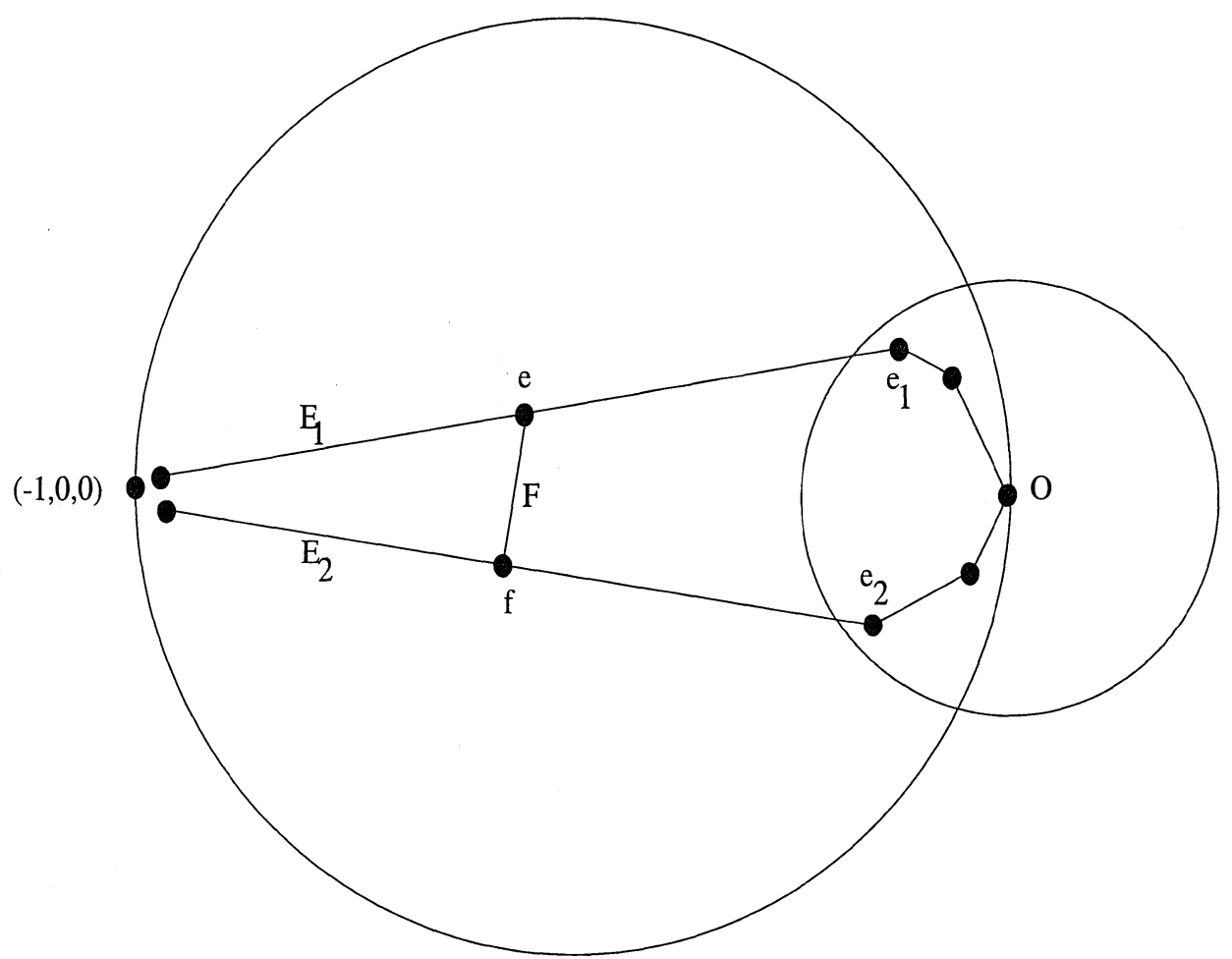

Figure 4.1.

We now show how to use Lemma 4.3 to establish the main result in this paper. By the remark following the proof of Lemma 4.1, we can assume our graph is embedded without affecting the validity of our "cut-and paste" argument described below. This means we can think of a standard lift as a geodesic-120 embedding of $S^{1}$.

Theorem 4.4. For each integer $n>1$ there exists an $R_{n}>0$ such that if $M$ is a closed, connected, hyperbolic 3-manifold and $\operatorname{Rank} \pi_{1}(M)=n$, then $\operatorname{inj}(M)<R_{n}$. 
Proof of Main Theorem. Given an integer $n>1$, we will show that there exists $R_{n}>0$ such that if a closed hyperbolic 3-manifold $M$ has Rank $\pi_{1}(M)=n$ and $\operatorname{inj}(M) \geq R_{n}$, then $\pi_{1}(M)$ is a free group. This will establish the theorem since the fundamental group of such a manifold cannot be a free group. Fix $n>0$. We shall use the notation $L(k)$ as in the statement of Lemma 4.3. Notice that we may choose $R>0$ so that if $n=\operatorname{Rank}\left(\pi_{1}(M)\right)$ and $\operatorname{inj}(M)>R$ then the following holds: if $f: \Gamma \longrightarrow M$ is a minimal length carrier graph, then every standard lift for kernel $\left(f_{*}\right)$ has two edges of length at least $L=L(3(n-1))$. Set $R_{n}=\max (R,(3 n-3) L(3 n-4))$. Thus, suppose $M$ has $\operatorname{inj}(M)>R_{n}$ and Rank $\pi_{1}(M)=n$. Let $h$ be a standard lift for kernel $\left(f_{*}\right)$ to $\mathbb{H}^{3}$. As in the proof of lemma 3.0, $h$ is a Geodesic-120 path through 0 with $H=h\left(S^{1}\right)$ contained in the horoball $\mathcal{W}$. Also, using the remark following Definition 2.5, we assume $h$ is an embedding of $S^{1}$. Notice that there are two edges $A$ and $B$ in $H$ with $|A|,|B|>L(3 n-3)$ and at most $3 n-3$ edges in the segment joining them through 0 . Consider the Geodesic-120 path $g$ defined by $A, B$ and the segment of $h$ containing 0 which joins them. By the proof of Lemma 3.0, since $|A|$ and $|B|$ are greater than $L(1), g$ is not a closed loop. Thus, $g$ satisfies the hypothesis of Lemma 4.3. Therefore, the assumption that $H$ is contained in $\mathcal{W}$ means there exist edges $E_{1}, E_{2}$ in $G=g([0,1])$ which satisfy $2 .(i)-2 .(i v)$ in Lemma 4.3. Let $e_{1}, e_{2}$ denote the respective endpoints of $E_{1}, E_{2}$ contained in the segment of $g$ which joins $E_{1}$ to $E_{2}$. This segment contains 0 . Moreover, since $H$ is contained in $\mathcal{W}$, the proof of Lemma 4.3 shows that we may assume that $E_{1}$ and $E_{2}$ are innermost with respect to 0 in the following sense: There is an integer $i>0$ with $\left|E_{1}\right|,\left|E_{2}\right|>L(i)$ and $e_{1}, e_{2} \in \bar{B}(0, i L(i-1))$ where $i L(i-1)<(3 n-3) L(3 n-4)$. To see this, note that if no such $i$ exists, then we may repeat the inductive argument in the proof of Proposition 4.2 to obtain a edge $E$ incident at 0 with $|E|>L(0)$. This contradicts the assumption that $H \subset \mathcal{W}$. 


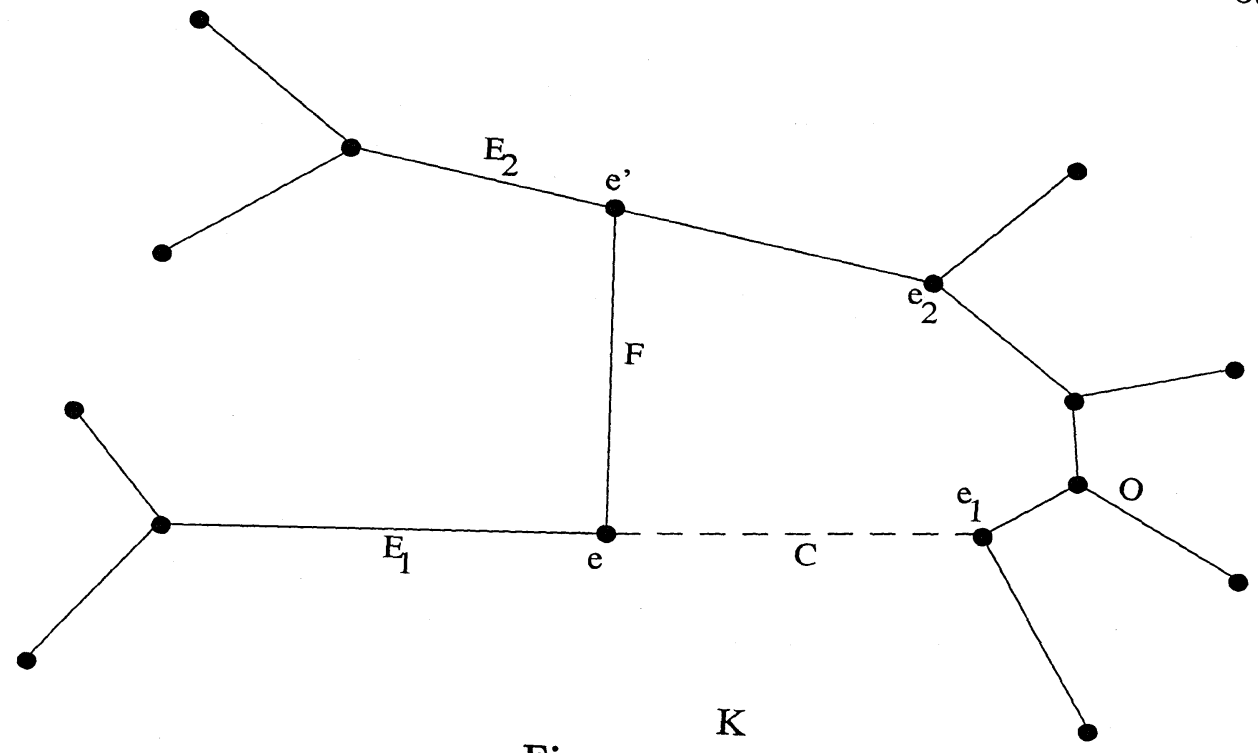

Figure 4.2.

Refer to Figure 4.2. We complete the proof using the arc $F$ joining $E_{1}$ to $E_{2}$ provided by Lemma 4.3. We let $e \in E_{1}$ and $e^{\prime} \in E_{2}$ denote the endpoints of $F$. Let $C$ denote the subarc of $E_{1}$ which joins $e_{1}$ to $e$. We wish to show that we can remove $C$ and attach $F$ such that the projection to $M$ gives a shorter carrier n-graph. Let $\rho: \mathbb{H}^{3} \longrightarrow M$ denote the universal cover of $M$. We first show that the modified image $(f(\Gamma)-\rho($ int $(C))) \cup \rho(F)$ defines a map of an $n$-graph into $M$. We can work with the image $f(\Gamma)$ since $\Gamma$ is embedded in $M$. Now, $\rho\left(e_{1}\right)$ is a vertex of $f(\Gamma)$. By removing $\rho(\operatorname{int}(C))$, we convert $\rho\left(e_{1}\right)$ into a bivalent vertex. Hence, we can amalgamate the remaining two edges incident at $\rho\left(e_{1}\right)$ into a single edge. This removes the vertex $\rho\left(e_{1}\right)$. We then view $\rho\left(\left(E_{1}-\operatorname{int}(C)\right) \cup F\right.$ as a single edge so that $\rho\left(e^{\prime}\right)$ becomes a trivalent vertex. This new subset of $M$ is an embedded, therefore, $f^{\prime}: \Gamma^{\prime}$ graph $f^{\prime}: \Gamma^{\prime} \longrightarrow M$ with the same number of vertices as $\Gamma$; Therefore, we need only show that $\Gamma^{\prime}$. Note also that $\ell\left(f^{\prime}\left(\Gamma^{\prime}\right)\right)<\ell(f(\Gamma))$. carrier $n$-graph $f: \Gamma \longrightarrow M$. Let $P$ carries $\pi_{1}(M)$. Consider again the joining $e_{2}$ to $e_{1}$ together with the subar ote the path defined by the edges then to show that $P$ can be used subarc of $E_{2}$ joining $e^{\prime}$ to $e_{2}$. The idea is if we show we have not cut used to replace $C$ in any loop of $\Gamma$. This follows $x_{0} \in f(\Gamma)-\left(\rho\left(E_{1}\right) \cup \rho\left(E_{2}\right)\right)$ and let $y_{0} \in f$ by removing $C$. Choose a basepoint $f_{*}$ is an epimorphism, there exists $[\beta] \in f^{-1}\left(x_{0}\right)$. Let $[\alpha] \in \pi_{1}\left(M, x_{0}\right)$. Since shall construct a corresponding loop $\beta^{\prime} \in \pi_{1}\left(\Gamma, y_{0}\right)$ with $f_{*}([\beta])=[\alpha]$. We from 2.(i) through 2.(iv) of Lemma $\beta^{\prime}$ in $\Gamma^{\prime}$ so that $f_{*}^{\prime}\left[\beta^{\prime}\right]=[\alpha]$. It follows 
which joins $E_{1}$ to $E_{2}$ has length less than both $E_{1}$ and $E_{2}$. Therefore, none of these edges projects to $\rho\left(E_{1}\right)$ or to $\rho\left(E_{2}\right)$. Now suppose $\rho\left(E_{1}\right)=\rho\left(E_{2}\right)$. We must have $\rho\left(e_{1}\right) \neq \rho\left(e_{2}\right)$ since otherwise $d\left(e_{1}, e_{2}\right)<(3 n-3) L(3 n-4)$ implies the geodesic edge with endpoints $e_{1}$ and $e_{2}$ projects to a nontrivial loop in $M$ of length less than $R_{n}$. This guarantees that the arc of $E_{2}$ joining $e^{\prime}$ to $e_{2}$ does not project to $\rho(C)$. Thus, $\rho(C)$ is not a subset of $\rho(P)$. In particular, $\rho(F \cup P)$ and $\rho(C)$ are paths in $M$ that are homotopic with endpoints fixed. These facts imply that if we imagine $\beta$ as a directed edge path in $\Gamma$, we can use the word for $\beta$ to build $\beta^{\prime}$ in $\Gamma^{\prime}$. That is, $\rho\left(E_{1}\right)$ corresponds to an edge $W_{1}$ in $\Gamma$ and $\rho((E-1-\operatorname{int}(C)) \cup F \cup P)$ corresponds to an edge path $W_{2}$ in $\Gamma^{\prime}$. In the word for $\beta$, replace $W_{1}\left(\right.$ resp. $\left.W_{1}^{-1}\right)$ by $W_{2}$ (resp. $W_{2}^{-1}$ ). This gives a loop $\beta^{\prime}$ in $\Gamma^{\prime}$. By construction, we may homotop $f \beta$ to $f^{\prime} \beta^{\prime}$ fixing $x_{0}$, which shows that $f_{*}^{\prime}$ is onto. This completes the proof of the main theorem.

Corollary 4.5. Given a closed hyperbolic 3-manifold $M$ and an integer $n>$ 0 , there exists a finite sheeted cover

$$
p: \tilde{M} \longrightarrow M
$$

with $\operatorname{Rank} \pi_{1}(\tilde{M}) \geq n$.

Proof. Given $M$ and $n>0$, let $\tilde{M}_{0}$ be a covering space of $M$. Suppose we have Rank $\pi_{1}\left(\tilde{M}_{0}\right)<n$. Then Theorem 4.3 shows $\operatorname{inj}\left(\tilde{M}_{0}\right)<R_{n} . \tilde{M}_{0}$ has finitely many closed geodesics $\gamma_{1}, \ldots, \gamma_{k}$ of length less than $R_{n}$. By residual finiteness, there exists a a covering space $\tilde{M}$ of $\tilde{M}_{0}$ such that the loops $\gamma_{1}, \ldots, \gamma_{k}$ do not lift. This implies $\operatorname{inj}(\tilde{M})>R_{n}$ so that $\operatorname{Rank} \pi_{1}\left(\tilde{M}_{0}\right) \geq n$.

Acknowledgements. The author thanks Professor Daryl Cooper for his encouragement and for many helpful conversations.

\section{References.}

[C] D. Cooper, The Volume of a Closed Hyperbolic 3-Manifold is Bounded by $\pi$ Times the Length of any Presentation of its Fundamental Group, Proceedings of the American Mathematical Society, 127(3) (1999), 941-942. 
[DC] M. Do Carmo, Riemannian Geometry, Birkhauser, Boston, 1993.

[E] P. Eberlein, Structure of Manifolds of Nonpositive Curvature, in 'Global Geometry and Global Analysis 1984,' Proceedings 1984, D. Ferus et.al. editors, Lecture Notes in Math., 1156, Springer, New York, (1985), 86-153.

[G] M.L. Gromov, Hyperbolic Groups, in 'Essays in Group Theory,' S. Gersten, MSRI Publications, 8, Springer, New York, (1987), 75-263.

[M] W. Massey, Algebraic Topology: An Introduction, Harbrace, New York, 1967.

[Pr] A. Preissman, Quelques proprietes globales des espaces de Riemann, Comm. Math. Helv., 15 (1943), 175-216.

[Th] W.P. Thurston, The Geometry and Topology of Three-Manifolds, Princeton University, 1979.

[W] M. White, A Diameter Bound for Closed Hyperbolic 3-Manifolds, preprint.

Department of Mathematics

California Polytechnic State University

SAN LUIS OBISPo, CA 93407

E-mail address: mewhite@calpoly.edu

Received April 21, 2000. 